\title{
MacArthur's Consumer-Resource Model
}

\author{
Peter Chesson \\ Department of Zoology, Ohio State University, \\ 1735 Neil Avenue, Columbus, Ohio 43210
}

Received July 19, 1989

\begin{abstract}
MacArthur's consumer-resource model is reviewed and new ways of understanding it are presented. Statistical measures of association between the utilization functions of different species are developed to show how coexistence conditions can be expressed in simple and understandable ways without the need to introduce strong symmetry assumptions. It is hoped that this new analysis will encourage both the use of the model in its full form without special simplifying assumptions, and the development of competition models of similar biological richness but different basic assumptions. 1990 Academic Press, Inc.
\end{abstract}

\section{INTRODUCTION}

Recently, several authors have discussed the prominence of the idea of competition in the history of community ecology (Schoener, 1982; Grant, 1986; Roughgarden, 1989). During much of this history, the LotkaVolterra competition equations provided theoretical guidance, but an important question was how could the parameters of the equations, especially the competition coefficients, be understood and measured empirically? Answers to this question were put forward in several articles by MacArthur and Levins. The first of these (MacArthur and Levins, 1967) introduced the concept of a utilization function and derived a formula for competition coefficients by heuristic arguments. A more elaborate formula with a firmer foundation was presented briefly in MacArthur (1969) and discussed fully in MacArthur (1970). This last article, which I find most enduring, was the first article of the first issue of Theoretical Population Biology. Its contributions are the subject of this paper.

The importance of MacArthur (1970) lies in its explicit analysis of a system of consumers interacting indirectly by exploiting common resources. The article has several sources of novelty. First, it made use of different timescales for the dynamics of consumers and resources. The Lotka-Volterra equations do not represent resources explicitly, and by exploiting these different timescales MacArthur showed how resources could be included in the Lotka-Volterra competition equations. The 
second source of novelty was a minimization principle, which is related to the use of Lyapunov functions for demonstrating global stability, although the latter does not seem to have been MacArthur's goal. The work, however, is mostly remembered for its formulae for competition coefficients.

These formulae seemed to provide a way of measuring niche overlap that might represent the actual effect of resource sharing on interactions between consumers. Soon after, Robert May, in collaboration with MacArthur (May and MacArthur 1972), coupled these formulae with ideas on the possible effects of a stochastic environment, and it appeared to many that the problem of community structure (an explanation for the numbers and relationships among competitors in natural environments) might have been solved. The reasons for failure of the theory to live up to this expectation have been extensively examined and debated elsewhere (see, e.g., Roughgarden 1989). It is not the purpose of this article to review this discussion. It is instead written with the conviction that a great deal of useful and robust theoretical work was in fact achieved at that time, and that its failings have more to do with overly ambitious expectations from distorted versions of the theory, than with any fundamental error in the theory itself.

For most users, MacArthur's competition coefficient formulae were too complicated to be implemented in practice. For empirical work, special assumptions were generally necessary before the formulae could be applied (Schoener, 1974a). Similar assumptions were often made for further theoretical development (e.g., May and MacArthur, 1972). As a consequence the full richness of the model presented by MacArthur was never explored.

This article proposes ways of understanding the original formulae without special assumptions. It is hoped that his may stimulate further theoretical study of the model and its generalizations in their full form. In addition, the development of alternative models of similar biological richness would be of great value.

\section{The Consumer-Resource Model}

MacArthur (1970) proposed the following equations for the percapita growth rate of a consumer species $i$ :

$$
\frac{1}{X_{i}} \cdot \frac{d X_{i}}{d t}=b_{i}\left(\sum_{l=1}^{m} c_{l l} w_{l} R_{l}-m_{i}\right) .
$$

Here, $X_{i}$ is population density; $R_{t}$ is the population density of resource species $l$, which in these equations is probably best thought of as a food 
resource; $w_{l}$ is the value of one unit of resurce species $l$ to the consumer; $c_{i l}$ is the rate at which consumer species $i$ captures resource $l$ per unit abundance of resource $l ; m_{i}$ is the total value of resource that must be harvested per capita for the growth rate to be exactly 0 . I shall refer to $m_{i}$ as the maintenance requirement, but we must remember that it includes all forms of loss from the system including death as well as physiological maintenance energy. Finally, $b_{i}$ is a factor converting the resource excess into the per capita growth rate.

The equation for resource dynamics has logistic population growth reduced according to consumer-imposed mortality:

$$
\frac{1}{R_{l}} \cdot \frac{d R_{l}}{d t}=r_{l}\left(1-\frac{R_{l}}{K_{l}}\right)-\sum_{i=1}^{n} c_{i l} X_{i}
$$

Properties of the full system of Eqs. (1) and (2) have been studied by Case and Casten (1979), but MacArthur's analysis assumed a timescale difference between consumers and resources. Population dynamics of resources were assumed to be faster, which means that $r_{l}$ and $c_{i l}$ are of larger order than $b_{i} c_{i l} w_{l}$ and $b_{i} m_{i}$. As a consequence, $R_{l}$ in Eq. (1) can be approximated by its consumer-dependent equilibrium value derived by setting the RHS of Eq. (2) to zero. With this substitution, Eq. (1) takes the Lotka-Volterra form

$$
\frac{d X_{i}}{d t}=b_{i}\left(k_{i}-\sum_{j} a_{i j} X_{j}\right) X_{i},
$$

where the coefficients $a_{i j}$ are given by

$$
a_{i j}=\sum_{l} c_{i l} c_{j l} \frac{w_{l} K_{l}}{r_{l}}
$$

and

$$
k_{i}=\sum_{l} c_{i l} w_{l} K_{l}-m_{i}
$$

Equations (3) need not have a unique positive equilibrium value $\mathbf{X}^{*}=\left(X_{1}^{*}, \ldots, X_{n}^{*}\right)^{\prime}$. As we shall see, when a unique positive equilibrium exists, it is globally stable. It is obvious from (3) that a unique positive equilibrium can only exist if the matrix $A=\left(a_{i j}\right)$ is of full rank. This is so if and only if the rows of the matrix $C=\left(c_{i l}\right)$ are linearly independent. Now the $i$ th row of $C$ is the vector of per unit consumption rates of resources by consumer species $i$, and may be called the resource utilization function of species $i$ (according to the usage of MacArthur, 1970). Linear independence of utilization functions means that no utilization function of a 
species can be manufactured by combining linearly the utilization functions of other species. In particular, no two species can have proportional utilization functions.

Given linear independence, the equilibrium is defined by $\mathbf{X}^{*}=A^{-1} k$.

The required solution therefore exists if all the components of the RHS of (6) are positive.

The next innovation, reviewed recently by Case (1980), was MacArthur's demonstration that the evolution of these equations minimizes a certain quadratic form involving resource availability and resource supply in the presence of a unique positive equilibrium. This quadratic form was related to the function

$$
D\left(\mathbf{X}, \mathbf{X}^{*}\right)=\sum_{i=1}^{n} b_{i}^{-1}\left(X_{i}-X_{i}^{*}-X_{i}^{*} \ln \frac{X_{i}}{X_{i}^{*}}\right) .
$$

This function has all the required properties for a Lyapunov function for this system (Goh, 1977) provided only that its time derivative is everywhere negative except at the equilibrium point. This time derivative is easily shown to be equal to

$$
\begin{aligned}
\frac{d}{d t} D\left(\mathbf{X}, \mathbf{X}^{*}\right) & =-\sum_{i=1}^{n} \sum_{j=1}^{n} a_{i j}\left(X_{i}-X_{i}^{*}\right)\left(X_{j}-X_{j}^{*}\right) \\
& =-\sum_{l=1}^{n} v_{l}\left(\sum_{i=1}^{n} c_{i l}\left(X_{i}-X_{i}^{*}\right)\right)^{2}
\end{aligned}
$$

where $v_{l}=w_{l} K_{l} / r_{l}$. It is clear that this quadratic form is negative definite if and only if the utilization functions are linearly independent. Without this linear independence, the system would approach a linear manifold of neutrally stable equilibria rather than a point equilibrium. Linear independence is thus necessary for a unique globally stable equilibrium point. In addition, the rate at which the Lyapunov function decreases, and thus the rate of approach to equilibrium, is related to the degree of linear independence.

Linear independence has featured in the above discussion in several critical places. First, it is necessary for uniqueness of the equilibrium, and as we shall see below, the degree of linear dependence is involved in the breadth of the parameter space permitting the equilibrium to be positive. Finally, we have just seen that it appears in the rate of approach to equilibrium.

In the work following MacArthur (1970), and in a number of other articles about the same time, there was much focus on the amount of linear independence needed for a stable coexistence. Usually, it was phrased in terms of the degree of niche overlap necessary for coexistence (May, 1973). 
To examine the permissible niche overlap, rather strong assumptions were made, including particular shapes for utilization functions, equality of the $k_{i}$, and equality of the $v_{l}$. It might be argued following MacArthur (1972) that the $v_{l}$ can be absorbed into the utilization functions, but this underrates the significant biological information that the $v_{l}$ contain. In multispecies contexts it was frequently assumed that the utilization functions are of equal widths and arrayed linearly equidistant apart. As pointed out by Roughgarden (1989), many thought that such assumptions, imposed to make the theory more understandable and more easily analyzed, were in fact conclusions. For example, constant body size ratios (Simberloff and Boecklen, 1981) that were presumed to come from competition theory in fact were only loosely associated with assumptions of convenience and had nothing at all to do with the conclusions.

MacArthur's model, however, and the formulae derived from it can be interpreted intuitively via the statistical measures discussed below, without making assumptions of convenience. Very special assumptions do not seem necessary for a good intuitive appreciation of the model.

\section{Statistical. Mfasurfes of I infar Depfndfnce}

A suitable measure of the degree of linear independence that does not involve special assumptions is the minimum eigenvalue of the matrix $A$ divided by the minimum diagonal element of $A$. This measure has a maximum value of 1 , which is achieved when all rows of $C$ are orthogonal to each other; in other words, when no two species share resources. This measure of linear independence can be related to the rate of approach to equilibrium given by the Lyapunov function. It gives the minimum rate of approach with the given utilization functions compared with the minimum that would apply if the utilization functions of different species were orthogonal.

This suggested measure of linear independence is related to principal component analysis (Anderson, 1984; Ludwig and Reynolds, 1988), where linear dependences of the rows of a data matrix are of interest. The columns define observations of different individuals or samples and the rows correspond to different measurements on a set of individuals or samples. In this regard the coefficient $a_{i j}$ is analogous to a covariance. The main difference is that in principal component analysis, linear dependence is usually examined with the mean of a row as the origin. Here 0 is the origin.

The problem can be approached from the opposite direction by looking for measures of linear dependence. If we examine two species at a time, such a measure indicates the closeness of the utilization function to proportionality. 
Plotting one utilization function against another, as in Fig. 1, gives a visual impression of how close the points lie to a straight line through the origin. Fitting such a line using weighted least squares with weights $v_{1}$ gives the relationship

$$
c_{l l}-\frac{a_{i j}}{a_{i i}} c_{t h}+\varepsilon_{l},
$$

where $\varepsilon_{l}$ is the error in fitting the line and $a_{i j} / a_{i i}$ is the regression coefficient. The elements of the matrix $A$ thus naturally appear. The weights, $v_{l}$, used in this regression are biologically significant quantities. They measure importance of a resource in terms of its value, $w_{l}$, multiplied by $K_{l} / r_{l}$. The latter quantity combines the unexploited equilibrium abundance of the resource and its basic per unit productivity, $r_{l}$, which determines its ability to remain plentiful in the face of exploitation.

How do we assess the closeness of this fit, i.e., the closeness to proportionality? The usual answer is by $R^{2}$ or what we shall call $\rho^{2}$ here. It is defined by

$$
\rho=\frac{a_{i j}}{\sqrt{a_{i i} a_{j j}}} .
$$

Now $\rho^{2}$ is the proportion of the weighted sum of squares

$$
\sum c_{j l}^{2} v_{l}=\sum\left(\frac{a_{i j}}{a_{i i}} c_{i l}\right)^{2} v_{l}+\sum \varepsilon_{l}^{2}
$$

accounted for by the fit of $c_{j l}$ to $c_{i l}$, i.e.,

$$
\rho^{2}=\frac{\sum\left(\left(a_{i j} / a_{i i}\right) c_{i l}\right)^{2} v_{l}}{\sum c_{i l}^{2} v_{l}} .
$$

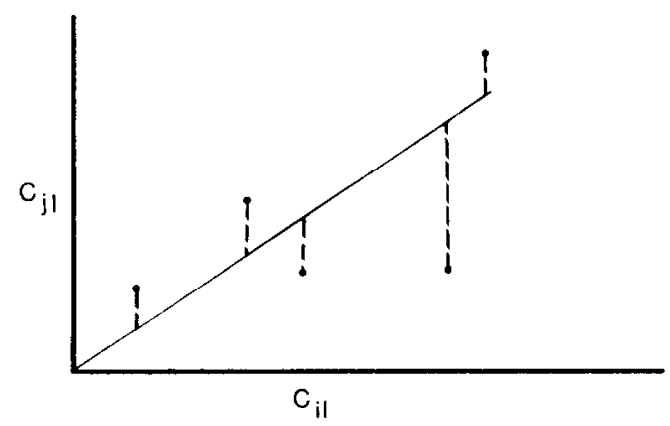

Fig. 1. The dots represent the utilization function, $c_{j l}$, of species $j$ plotted against the utilization function of species $i$. The solid line is the weighted least squares proportional fit of $c_{j l}$ to $c_{i l}$, and the vertical lines represent the deviations $\varepsilon_{l}$ from proportionality. 
Note that while this formulation is asymmetric in $i$ and $j$, the actual value of $\rho$ is symmetric in $i$ and $j$ (formula (10)); i.e., it does not matter whether we consider a weighted least squares fit of $j$ on $i$ or $i$ on $j$.

The most useful aspect of $\rho$ is that it can be related directly to existence of a positive equilibrium in a two-species system. If $\rho=1$, the utilization functions are proportional and there is no unique solution; however, if $\rho<1$, examination of Eq. (6) shows that a positive equilibrium exists if and only if

$$
\theta \rho<\frac{k_{i}}{k_{j}}<\frac{\theta}{\rho}
$$

where the scaling constant $\theta=\sqrt{\left(a_{i i} / a_{i j}\right)}$ measures the relative magnitudes of the utilization functions. This shows that with very small values of $\rho$ there is a broad range of possible values of the ratio of $k_{i}$ and $k_{j}$ consistent with coexistence. However, if $\rho$ is close to $1, k_{i} / k_{j}$ must be very close to 1 for coexistence to be possible. These relationships are represented in Fig. 2, with a $\log$ scale for $k_{i} / k_{j}$ to give a symmetrical diagram.

The analysis here is closely related to that given by May (1973, formula (6.25)), with the difference here being simply that no symmetry assumptions have been made, and the interpretation is directly in terms of measures of proportionality rather than in terms of spacing of utilization functions on a recource axis.

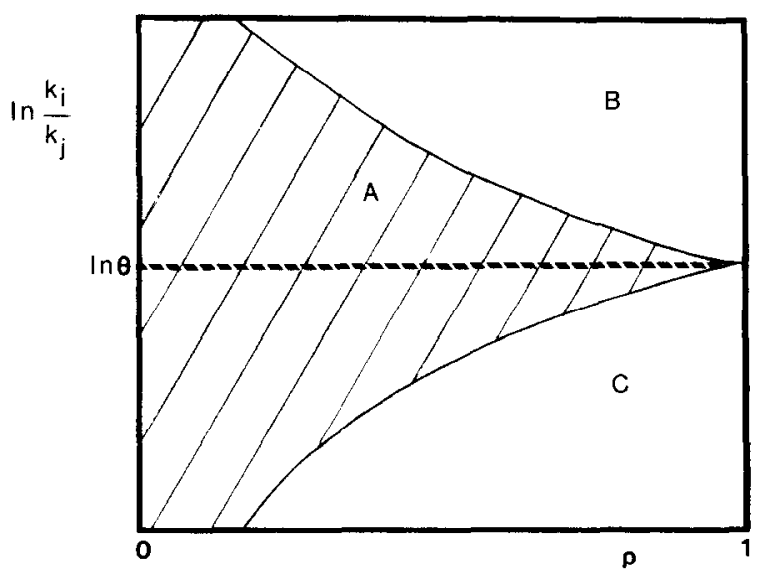

FIG. 2. Coexistence regions in the two-consumer version of MacArthur's model in terms of the ratio $k_{i} / k_{j}$, the measure of proportionality $\rho$, and the relative magnitudes of the utilization functions $\theta$. A is the coexistence region. In $B, j$ is excluded, and in $C, i$ is excluded. 
Further insight is available if we delve into the formula for $k_{i}$. Defining $h_{i}=\sum_{l} c_{i l} w_{l} K_{l}$, we have from (5)

$$
k_{i}=h_{i}-m_{i} \text {. }
$$

The quantity $m_{i}$ is the maintenance requirement, and $h_{i}$ is the amount of resource that would be harvested by each individual if there were no competition (and each resource were therefore permanently at carrying capacity).

The ratio of $h_{i}$ to $h_{j}$ takes into account the relative magnitudes of the utilization functions of the two species as measured by $\theta$. Indeed, when $\rho$ is near $1, h_{i} / h_{j}$ will be near $\theta$. Therefore, requiring the ratio of $k_{i}$ to $k_{j}$ to be near $\theta$ is equivalent to requiring the ratio of the maintenance requirements to be approximately equal to $\theta$. Thus, maintenance requirements and harvesting rates must be approximately proportional if the species are to coexist with $\rho$ near 1 . Such proportionality is perhaps a consideration in physiological ecology. Maintenance energy might be determined allometrically (Peters, 1983); if harvesting rate is determined similarly, then this requires identical allometric exponents for harvesting rates and maintenance requirements.

It must be kept in mind, however, that the maintenance requirement $m_{i}$ involves all forms of loss from the population in addition to metabolic maintenance energy including death of individuals by all causes. Thus, if one species is more subject to predation and disease than another, its maintenance requirement will be increased in comparison to the other species. For coexistence, a value of $\rho$ near 1 means that the ecological differences between species that could lead to differences in maintenance requirements are sharply restricted.

\section{GeNeralizations}

A significant weakness of MacArthur's consumer-reource model is the assumption that there are no interactions between resources except the indirect interaction that comes about from the numerical response of consumers to resources. The use of the logistic growth model implies that resources are biological. Since in practice the resources are often similar species, they might interact through competition, and in general, predatorprey interactions or mutualism can be expected. In other cases, sizes or stages of single species may be treated differently by consumers, so that multistage resource dynamics should be included. Finally, when resources are defined by places or times of foraging on the same species or same set of species, movements of resource species in space and connections over time need to be considered, too. 
MacArthur (1970) made an important start on this collection of possibilities by permitting resource dynamics to take the general form

$$
\frac{1}{R_{l}} \cdot \frac{d R_{l}}{d t}=r_{l}\left(1-\frac{1}{K_{l}} \sum_{u=1}^{m} b_{l u} R_{u}\right)-\sum_{i=1}^{n} c_{i l} X_{i} .
$$

This allows any relationship among the resources compatible with a linear per capita growth rate. Making the same timescale assumptions as before, the Lotka-Volterra equation (3) again results, but the formula for the $a_{i j}$ is now

$$
a_{i j}=\sum_{p=1}^{m} \sum_{q=1}^{m} c_{i p} v_{p q} c_{j q}
$$

where the weight matrix $V=\left(v_{p q}\right)$ is defined as

$$
V=W B^{-1} U
$$

with $W=\operatorname{diag}\left(w_{l}\right), B=\left(b_{l u}\right)$ and $U=\operatorname{diag}\left(K_{l} / r_{l}\right)$. Note that this differs from MacArthur (1970), due to a misidentification of subscripts.

The form of the matrix $V$ is important for further development. If $V$ is a positive definite symmetric matrix, then $A$ remains symmetric and the previously suggested measures of linear independence and proportionality are still applicable. The main difference is that the weights $v$, used before have been replaced by the weight matrix $V$ and are not so simply interpreted. For the measure $\rho$, positive definiteness of $V$ can be relaxed to simply that $A$ be a positive definite symmetric matrix, which can occur without $V$ s being positive definite. A little algebra shows that for an arbitrary constant $b$,

$$
\begin{aligned}
& \sum_{p, q}\left(c_{j p}-b c_{i p}\right) v_{p q}\left(c_{j q}-b c_{i q}\right) \\
& =\left(a_{i j} / a_{i i}-b\right)^{2} a_{i i}+\sum_{p, q} \varepsilon_{p} v_{p q} \varepsilon_{q},
\end{aligned}
$$

where $\varepsilon_{l}=c_{j l}-\left(a_{i j} / a_{i i}\right) c_{i l}$. This means that the quadratic form on the LHS is minimized by setting $b=a_{i j} / a_{i i}$. Thus, using this quadratic form to judge fit to a straight line through the origin, the best fit is obtained with $b=a_{i j} / a_{i i}$. The interpretation of $\rho$ as defining the closeness of a fit to a straight line is still applicable. Setting $b=0$ in (18) shows that $\rho^{2}$ measures the fraction of

$$
\sum_{p, q} c_{j p} v_{p q} c_{i q}=a_{j j}
$$

attributable to the weighted regression through 0 of $c_{j l}$ on $c_{i l}$. 
When $A$ is not positive definite, we enter uncharted territory. In particular, the two-species coexistence criterion, (13), no longer applies.

\section{Discussion}

Recently there has been a strong appreciation of the need to study competition models that cannot be represented in the Lotka-Volterra form (Turelli, 1981; Schoener, 1982; Chesson, 1988; Roughgarden, 1989). However, it would be wrong to think that Lotka-Volterra models have no further instructional value. MacArthur's resource-competition model, which can be reduced to Lotka-Volterra form, has a richness of biological detail that deserves further study. Within the context of substitutable resources (Tilman, 1982) it permits arbitrary complexity of resource use and specifies in a detailed quantitative way how resource use differs among species. The development of other ecological models with similar detail, but without Lotka-Volterra assumptions, would be useful to see in what ways the results of the model generalize, and also to provide alternatives to it. Schoener (1974b) has provided a beginning to such a program.

The results in the section on generalizations, above, show that the simplest results from MacArthur's model, interpreted naively, do not generalize. In particular, the formula (4) for the $a_{i j}$ is incorrect when the model is generalized. However, interpreted more broadly, the results clearly do generalize in the sense that the formula (4) for the $a_{i j}$ is a special case of formula (16). This does not make formula (4) wrong, only restricted in its application, like all formulae. Thus, further exploration of models, even Lotka-Volterra modcls, will show the conclusions of MacArthur's model to be wrong in the narrow sense, but they will be correct in a broader sense.

For further illustration consider the remark of Case and Casten (1979) that the simple global stability properties of MacArthur's model might be replaced by multiple domains of attraction in the presence of some kinds of interference competition. To find a generalization of MacArthur's results on global stability one might consider interference and exploitation simultaneously in a model and ask whether exploitative competition as defined in MacArthur's model has a general tendency to reduce the number of domains of attraction.

Research interest in MacArthur's model should extend beyond attempts to generalize it, for there is much still be learned from the model in its original form. As has been emphasized, most studies of MacArthur's model proceed as if the weighting terms $v_{t}$ in the $a_{i j}$ did not exist. Moreover, strong symmetry assumptions on the relationships of among utilization 
functions and among resurces are often imposed; however, Roughgarden (1979) has discussed some variations on strict symmetry.

As illustrated by Spiller's (1986) field study, the terms, $v_{l}$, incorporating resource value and resource productivity have important information. For example, making one of these much larger than others reduces linear independence for it places great emphasis on just one resource. Thus, coexistence is much more difficult. In some cases, the $v_{1}$ could be manipulated experimentally in nature, and such manipulations could lead to new tests of the model and new ways of exploring competition.

Does the model predict patterns of size differences among competing species, as have often been sought in competition studies (e.g., Schoener, 1984)? In the absence of many special assumptions, such results are not predicted by the model (Roughgarden, 1989). Indeed, simple overall patterns applicable broadly are unlikely to result. The patterns that are produced will depend on information on resources, as incorporated in the much neglected $v_{1}$, in addition to the possible forms that the utilization functions might take.

Any test of such patterns will likely involve information of a different and more detailed nature than ecologists have been accustomed to. There is no reason to believe that such a test would be intractable. For example, I have mentioned above that the $v_{l}$ might be subject to experimental manipulation. However, it also might be concluded that in some systems community dynamics cannot be adequately investigated with current technology. This is a better conclusion than an affirmation or rejection of competition based on a seriously oversimplified mathematical or verbal model.

There two other useful areas of exploration of MacArthur's model that I would like to mention. First, the statistical measure of linear dependence derived here needs to be extended to the multiconsumer case, and more generally to the case of interacting resources. Second, the interacting resource model needs further exploration because very few conclusions are available from it as yet. Because interacting resources might lead to an insuperable degree of complexity for study in nature, it would be useful to know when such interactions can safely be ignored or got around by suitable aggregation of resources. For example, a guild of competing resources in some circumstances could be treated like a single resource.

The statistical measures derived here are intended to show that MacArthur's model, as rich and detailed as it is, can be reduced to intuitive tractability without making special assumptions. Measures of a similar nature but applied to competition in a stochastic environment are discussed by Chesson and Huntly (1988). Given the complexity of the natural world, it seems not unreasonable to propose that strong theoretical explanations of the functioning of natural communities might well 
generally involve statistical measures summarizing the diverse effects of the many species, processes, microhabitats, etc. Such statistical measures would be derived from models of ecological processes.

\section{ACKNOWLEDGMENTS}

I am grateful for Tom Schoener's careful reading of the manuscript, which helped remove several ambiguities. I thank Robert MacArthur for the many gems that I have discovered in his work while writing this article. This work was supported by NSF Grant BSR 8615028.

\section{REFERENCES}

ANDERSON, T. W. 1984. "An Introduction to Multivariate Statistical Analysis," 2nd ed., Wiley, New York.

CASE, T. J. 1980. MacArthur's minimization principle: A footnote, Amer. Nat. 115, 133-149.

CASE, T. J., AND CASTEN, R. G. 1979. Global stability and multiple domains of attraction in ecological systems, Amer. Nat. 113, 705-714.

Chesson, P. L. 1988. Interactions between environment and competition: How fluctuations mediate coexitence and competitive exclusion, in "Community Ecology" (A. Hastings, Ed.), Lecture Notes in Biomathematics, Vol. 77, pp. 51-71, Springer-Verlag, Berlin/New York.

Chesson, P., and Huntly, N. 1988. Community consequences of life-history traits in a variable environment, Ann. Zool. Fenn. 25, 5-16.

GoH, B. S. 1977. Global stability in many species systems, Amer. Nat. 111, 135-143.

Grant, P. R. 1986. Interspecific competition in fluctuating environments, in "Community Ecology" (J. Diamond and T. J. Case, Eds.), pp. 173-191, Harper \& Row, New York.

LudwiG, J. A., AND ReYnolds, J. F. 1988. "Statistical Ecology," Wiley, New York.

MacArthur, R. H. 1969. Species packing and what competition minimizes, Proc. Natl. Acad. Sci. U. S. A. 64, 1369-1371.

MacArthur, R. H. 1970. Species packing and competitive equilibria for many species, Theor. Pop. Biol. 1: 1-11.

MacArthur, R. H. 1972. "Geographical Ecology: Patterns in the Distribution of Species," Princeton Univ. Press, Princeton, NJ.

MacArthur, R. H., AND Levins, R. 1967. The limiting similarity, convergence and divergence of coexisting species, Amer. Nat. 101: 377-385.

MAY, R. M. 1973. "Stability and Complexity in Model Ecosystems," Princeton Univ. Press, Princeton, NJ.

MAY, R. M., AND MacArThUR, R. H. 1972. Niche overlap as a function of environmental variability, Proc. Natl. Acad. Sci. U.S.A. 69: 1109-1113.

Peters, R. H. 1983. "The Ecological Implications of Body Size," Cambridge Univ. Press, Cambridge, U.K.

Roughgarden, J. 1979. "Theory of Population Genetics and Evolutionary Ecology: An Introduction," Macmillan, New York.

Roughgarden, J. 1989. The structure and assembly of communities, in "Perspectives in Ecological Theory" (J. Roughgarden, R. M. May, and S. A. Levin, Eds.), pp. 203-226, Princeton Univ. Press, Princeton, NJ.

SCHOENER, T. W. 1974a. Some methods for calculating competition coefficients from resource utilization spectra, Amer. Nat. 108, 332-340. 
Schoener, T. W. 1974b. Competition and the form of habitat shift, Theor. Pop. Biol. 6, $265-307$.

SCHOENER, T. W. 1982. The controversy over interspecific competition, Amer. Sci. 70, $586-595$.

SCHOENER, T. W. 1984. Size differences among sympatric bird-eating hawks: A worldwide survey, in "Ecological Communities: Conceptual Issues and the Evidence" (D. R. Strong, Jr., D. Simberloff, L. G. Abele, and A. B. Thistle, Eds). pp. 254-281, Princeton Univ. Press., Princeton, NJ.

Simberloff, D., AND BoECKLEN, W. 1981. Santa Rosalia reconsidered: Size ratios and competition, Evolution 35, 1206-1228.

SPIller, D. A. 1986. Consumptive-competition coefficients: An experimental analysis with spiders, Amer. Nat. 127, 604 614.

Tilman, D. 1982. Resource competition and community structurc, Princeton Univ. Press, Princeton, NJ.

TURELLI, M. 1981. Niche overlap and invasion of competitors in random environments. I. Models without demographic stochasticity, Theor. Pop. Biol. 20, 1-56. 